\title{
TOC: Localizing Wireless Rechargeable Sensors with Time of Charge
}

\author{
Yuanchao Shu*, Peng Cheng ${ }^{* \dagger}$, Yu Gu ${ }^{\dagger}$, Jiming Chen* and Tian $\mathrm{He}^{\ddagger}$ \\ * State Key Laboratory of Industrial Control Technology, Zhejiang University, China \\ $\dagger$ Singapore University of Technology and Design, Singapore \\ $\ddagger$ Dept. of Computer Science and Engineering, University of Minnesota, USA
}

\begin{abstract}
Wireless rechargeable sensor network is a promising platform for long-term applications such as inventory management, supply chain monitoring and so on. For these applications, sensor localization is one of the most fundamental challenges. Different from traditional sensor node, wireless rechargeable sensor has to be charged above a voltage level by the wireless charger in order to support its sensing, computation and communication operations. In this work, we consider the scenario where a mobile charger stops at different positions to charge sensors, and propose a novel localization design that utilizes the unique Time of Charge (TOC) sequences among wireless rechargeable sensors. Specifically, we introduce two efficient region dividing methods, Inter-node Division and Inter-area Division, to exploit TOC differences from both temporal and spatial dimensions to localize individual sensor nodes. To further optimize the system performance, we introduce both an optimal charger stop planning algorithm for single sensor case and a suboptimal charger stop planning algorithm for the generic multisensor scenario with a provable performance bound. We have extensively evaluated our design by both testbed experiments and large-scale simulations. The experiment and simulation results show that by as less as 5 stops, our design can achieve sub-meter accuracy and the performance is robust under various system conditions.
\end{abstract}

\section{INTRODUCTION}

Wireless Rechargeable Sensor Networks (WRSN) is an emerging technology which integrates sensing, communication and computation capabilities. Different from the traditional sensor nodes powered by batteries, wireless rechargeable sensor nodes gather their energy from the transmission of energy sources such as RFID readers. Given its small form factors and universal sensing capabilities, it is expected that wireless rechargeable sensor will be a promising platform for different applications such as warehouse inventory management [3], [16], supply chain monitoring [2], [19], authentication [24], [29] and so on.

For many of such applications, the locations of sensor nodes are required for them to function properly. For example, for warehouse inventory management or environmental monitoring, it is usually necessary to identify the location where sensor readings are originated from. In addition, some geographic routing protocols and network management optimization [13],

978-1-4799-3360-0/14/\$31.00 (C)2014 IEEE
[15] can only be implemented under the assumption that the location of each sensor is already known.

For traditional wireless sensor networks, there are numerous localization methods which utilize the arrival sequences of a specific signal source to localize sensors, e.g., Time of Arrival (TOA), Angle of Arrival (AOA), Time Difference of Arrival (TDOA) etc. [12]. Different from traditional approaches, in this work we exploit the unique wireless charging properties of the wireless rechargeable sensor nodes and propose the concept of Time of Charge (TOC), the time for a sensor node to be charged above its working threshold, to localize individual nodes in a WRSN.

In this paper, we consider the scenario that a mobile charger moves and stops at different locations to wirelessly charge nodes and obtain the time of charge (TOC) for nodes in its surrounding area. It typically refers to a warehouse or supermarket where patrol readers monitor status of goods (e.g. temperature, moisture) reported by attached wireless rechargeable sensors. The novel idea of TOC is to estimate each sensor's location by utilizing Time of Charge sequences from both temporal and spatial domain. Furthermore, TOC also optimizes the charger stop positions based on the estimated sensor locations.

TOC offers several unique benefits over traditional methods. Firstly, compared with traditional methods such as TOA, AOA and TDOA which are all rely on the instantaneous readings from a specific signal source, TOC is an accumulative reading, which is more robust and resilient to transient signal fluctuations. Secondly, compared with the range-based approach, TOC does not require additional costly hardware. It works with existing components of WRSN nodes such as differential comparators and real-time clock (RTC). Thirdly, compared with many range-free approaches, TOC only leverages the mobile charger in WRSN and does not require any anchor node, which reduces the infrastructure cost. Fourthly, TOC is only based on the fact that charging time monotonically increases when the distance increases and does not have any assumption on specific charging model, making it compatible with different types of wireless rechargeable sensor networks [22], [26] and designs in mobile sensor networks [1], [4], [10], [33].

This paper offers the following intellectual contributions: 


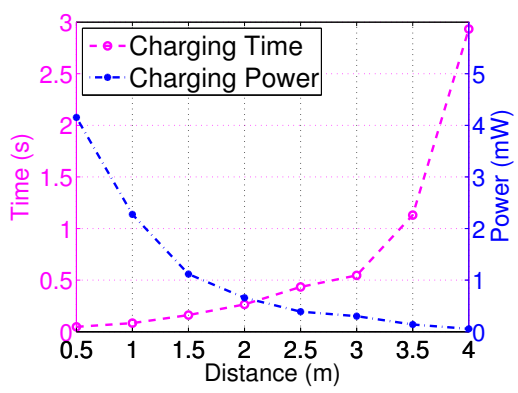

Fig. 1. Charging Power and Charging Time

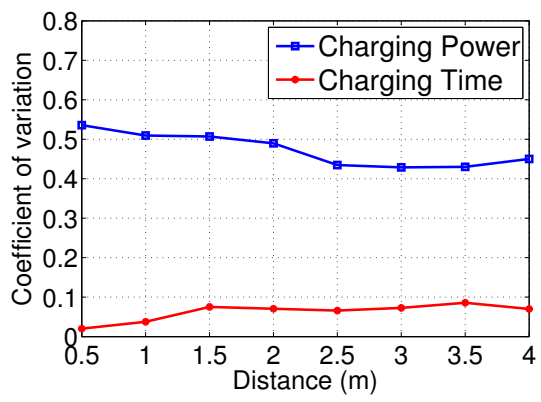

Fig. 2. Coefficient of Variation

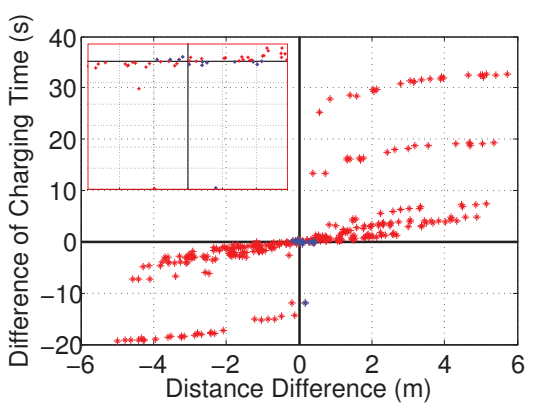

Fig. 3. Measurement Unreliability
- To the best of our knowledge, this is the first work designed for localizing wireless rechargeable sensor nodes based on the Time of Charge sequences. We identify the fundamental relationship between the charging time and the distance between wireless rechargeable sensor nodes and the charger, based on which we propose the Time of Charge sequence based localization design (TOC).

- We introduce two novel area division methods for localizing sensors, i.e., Inter-node Division and Inter-area Division, which exploit TOC differences among sensors from time and spatial domain respectively.

- We propose an optimal charger stop solution for locating a single sensor node. For the more general multi-sensor scenario, we introduce a sub-optimal solution with a provable approximation ratio.

- We have implemented our TOC design on a physical testbed. The experiment results show that by as less as 5 stops, we can achieve sub-meter localization accuracy for a wireless rechargeable sensor network.

The remainder of this paper is organized as follows. We introduce some preliminary knowledge of charging pattern in Section II. In Section III, we define the localization problem and propose the basic design of TOC. In Section IV, we propose the extended TOC algorithms. In Section V, we discuss the impact of occasional charging time flips and provide corresponding solutions. We extensively evaluate TOC through testbed experiments and large-scale simulations in Section VI. Most related work are discussed in Section VII. Finally, we conclude in Section VIII.

\section{Preliminary}

One of the most common wireless rechargeable sensor nodes is the Wireless Identification Sensing Platform (WISP) developed by Intel Research [22]. WISP is a fully-passive Ultra High Frequency (UHF) RFID tag that integrates a processor and several low-power sensors such as accelerometer and temperature sensors. Through its antenna, a WISP node accumulates energy from signals of nearby standard UHF RFID readers (the charger), and stores the harvested energy in its capacitor for communication and powering other components of the node.

For the charging process, the exact charging model is usually difficult to obtain due to factors such as polarization and antenna impedance. However, one of our major observations is that the charging power is typically negatively correlated to the relative distance between the wireless rechargeable node and the energy source, e.g. the charger. Many mathematical analysis and experimental results also prove there exists a negative correlations between the charging power and distance [11]. To verify this observation, we conduct a series of experiments of WISP with capacitor of $100 \mu F$ and a charger with transmission power of $30 \mathrm{dBm}$.

We first record how the charging power varies with the charging distance. As shown in Figure 1. It can be observed that the received charging power decreases monotonically with the charging distance. For different charging distances, we also record the charging time by which the voltage reaches $2 \mathrm{~V}$ from zero. As indicated in Figure 1, the charging time from 0 to $2 \mathrm{~V}$ increases monotonically with the increasing relative distance.

Thus, it can be concluded that despite of the difference among different charging models, there always exists a negative correlation between the charging power and distance. In another word, a positive correlation between the time of charging and distance. While it is costly to measure the exact charging power at the low-cost and tiny sensor node, we can alternatively compute the charging time by monitoring voltage of the capacitor by the available differential comparators and the real-time clock (RTC). Thus for our TOC design, we utilize the simple fact that the node with longer charging time is further away from the charger, the positive correlations between the charging time and charging distance to localize individual nodes.

Besides the ease of measurement, there are other benefits of utilizing TOC. For the existing sequence-based localization methods, such as TOA, AOA and TDOA, usually require highly accurate measurements. For example, TOA [23] needs precise measurements of time, and the AOA method [18] requires accurate measurement of angle. However for our TOC design, we utilize the accumulative charging process in a period of time and thus is more robust and resilient to the instantaneous fluctuations of wireless charging power.

To verify the stability and robustness of using time of charging over instantaneous metrics such as charging power, we conduct experiments on our WISP-based testbed. We randomly 
place 6 sensor nodes and a charger in an outdoor parking lot for multiple topologies and record charging curves of each node. Charging time and power are then calculated based on the charging curve. Figure 2 shows the coefficient of variation of charging power and charging time vs. different charging distances. From Figure 2 we see that the coefficient of variation of charging power is always higher than 0.4 whereas the coefficient of variation of charging time remains below 0.1 . Since the coefficient of variation is a normalized measure of dispersion of a probability distribution, results in Figure 2 validate the robustness of using time of charging over charging power.

Furthermore, in the experiment we also examine the occurrence frequency of occasional charging time fluctuations. During each stop of the charger, we calculate the difference of charging time as well as the difference of charging distance for all node pairs and map them in Figure 3. From Figure 3, we find that the majority of points located in the first quadrant and the third quadrant, which further proves the positive correlation between the charging time and distance. In Figure 3, points located in the second quadrant and the fourth quadrant are marked in blue (also shown in a zoomed-in figure in the upleft corner). From the results we can see that all blue dots located near axis $x=0$. It means that although charging time fluctuations occur occasionally, they are within a very narrow band. It is consistent with intuition that such fluctuations are more likely to happen when two nodes share the similar charging distances from the reader. Such a property makes it possible to directly filter out the fluctuated measurements even such measurement error happens occasionally. In Section V, we will discuss a simple yet effective solution to resolve such occasional charging time fluctuations.

\section{BASIC TOC DESIGN}

In this section, we elaborate the basic TOC design, which consists of Inter-node Division and Inter-area Division.

\section{A. Settings}

Consider $N$ wireless rechargeable nodes randomly deployed in an area of size $S$. We define the feasible region for each node $i$ as the minimal region, where node $i$ is guaranteed to be inside. At the beginning of the localization process, the feasible region of each target node is simply the whole region $S$. Moreover, in TOC, we set the center of gravity of the feasible region as the estimated location of the target node. Intuitively, it is necessary to narrow down the feasible region in order to obtain an accurate localization result.

To locate target nodes, one charger moves freely within the area. The charger is able to decide where to stop and turn its radio on to charge the nearby sensors. Each sensor responses to the charger once its voltage reaches a threshold. And each sensor will return to the fully discharged state before the next recharging due to the workload and highly limited energy capacity. For example, the discharge rate is around $10^{-3} \mathrm{~W}$ for a typical WISP node equipped with $10 \mu F$ capacitor, and the discharging time is around $100 \mathrm{~ms}$ [20], which is considerably short. We denote the charging time for reaching a fixed voltage threshold as the charging time for each node.

Generally, different charger stop positions make the charging time vary for each sensor. In this part, we show that even random stop of the charger could greatly reduce the feasible region of each sensor. In the next section, we will further discuss how to optimize the charger stop positions for better localization performance.

For the purpose of introducing the key idea, in this section we assume the TOC readings at individual nodes are strictly negatively correlated with their distances to the charger. In Section V, we will relax this assumption and discuss how to deal with occasional TOC reading flips in practice.

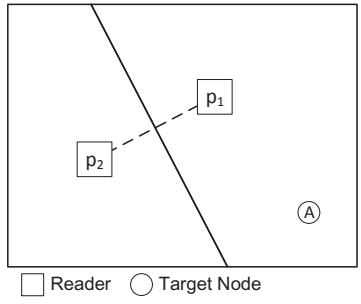

(a) Feasible Region after Two Stops of the Charger

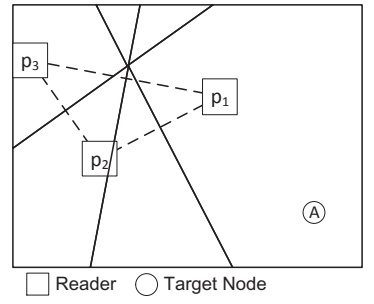

(b) Feasible Region after Three Stop of the Charger
Fig. 4. Inter-node Division

\section{B. Inter-node Division}

We first illustrate the idea of how to narrow down the feasible region of each node individually with continuous stops of the charger, which is named as Inter-node Division.

In Figure 4(a), target node A is to be localized, denoted by numbered circles. The charger randomly stops at two different positions, which are denoted by numbered squares, i.e., $p_{1}$ and $p_{2}$. Since node $\mathrm{A}$ is closer to $p_{1}$, it would have a shorter charging time for charger position $p_{1}$ than $p_{2}$. Therefore, we can infer that node A must lie on the right of the perpendicular bisector of these two stop positions.

Suppose the charger stops at another position, denoted by $p_{3}$ as in Figure 4(b). We can draw another two perpendicular bisectors, and further narrow down the feasible region (i.e. the shaded area) of node A.

Denote $t(A, p)$ as the time of charge for node $\mathrm{A}$ when the charger stops at position $p$, and $d\left(p_{i}, p_{j}\right)$ as the Euclidean distance between two points $p_{i}$ and $p_{j}$. This property can be summarized in the following lemma.

Lemma 3.1: Consider an arbitrary pair of charger stop positions, $p_{1}$ and $p_{2}$, and one node $A$. If $t\left(A, p_{1}\right) \leq t\left(A, p_{2}\right)$ then $d\left(A, p_{1}\right) \leq d\left(A, p_{2}\right)$, and vice versa.

It can be proved that, by Inter-node Division, all feasible regions remain as convex polygons. In addition, we notice that the increasing number of stop positions will generate exponentially increasing number of perpendicular bisectors. Specifically, the $n$-th stop of the charger generates $n-1$ 
perpendicular bisectors. And a total of $\frac{n(n-1)}{2}$ perpendicular bisectors will exist after $n$ times of stops, which will divide the original feasible region into $\frac{\left(n^{4}-2 n^{3}+3 n^{2}-2 n+8\right)}{8}$ pieces at most [25]. Thus it is expected that Inter-node Division will be very effective to narrow down the feasible regions of all sensors simultaneously. According to our simulations, even with random charger stops, the area of feasible region of one node can be narrowed down to $7.8 \%$ of the original region after only 5 stops of the charger.

\section{Inter-area Division}

In this part, we show how to further narrow down the feasible region obtained from Inter-node Division by utilizing the Time of Charge difference of each pair of sensors for the same charger position. The novel idea is that each node, which may not have been accurately localized, can help to narrow down the feasible regions of their peers.



(a) Case 1

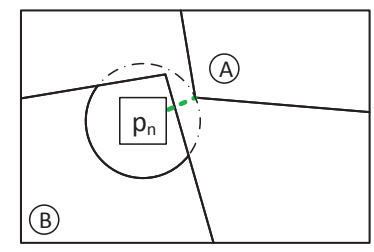

(b) Case 2
Fig. 5. Inter-area Division

We explain the idea by a simple example. Suppose node A and node $\mathrm{B}$ are distributed in an area with their initial feasible regions denoted by $R_{A}$ and $R_{B}$ respectively, as shown in Figure 5. The charger stops at $p_{n}$ to charge A and B simultaneously. First, if $p_{n}$ is within the feasible region of $\mathrm{A}$ as shown in Figure 5(a), as the charging time of $\mathrm{A}$ is shorter than that of $\mathrm{B}$, we can determine that the feasible region of $\mathrm{A}$ should be inside the circle centered at $p_{n}$ (i.e. the dash dotted line) with radius equal to the farthest distance between $p_{n}$ and $R_{B}$ (i.e. the dash line). However, if $p_{n}$ is within the feasible region of $\mathrm{B}$ as shown in Figure 5(b), as the charging time of $\mathrm{A}$ is still shorter than that of $\mathrm{B}$, we can infer that the feasible region of $\mathrm{B}$ should be outside the circle centered at $p_{n}$ with radius equal to the shortest distance between $p_{n}$ and $R_{A}$.

Denote $d_{\text {max }} / d_{\min }(\cdot, \cdot)$ represents the maximal/minimal distance function. The above properties are summarized in the following lemma.

Lemma 3.2: Consider an arbitrary pair of nodes, A and B with initial feasible region as $R_{A}$ and $R_{B}$ respectively. Suppose the charger stops at $p_{r}$ and the charging time of node $\mathrm{A}$ is less than that of node $\mathrm{B}$, then $\forall p_{A} \in R_{A}, d\left(p_{A}, p_{r}\right)<$ $d_{\text {max }}\left(R_{B}, p_{r}\right)$, and $\forall p_{B} \in R_{B}, d\left(p_{B}, p_{r}\right)>d_{\text {min }}\left(R_{A}, p_{r}\right)$.

To further understand Lemma 3.2, let us consider the example depicted in Figure 6(a). If we find that the charging time of node $\mathrm{B}$ is less than that of node $\mathrm{A}$ when the charger stops at the first position $p_{1}$, then based on Lemma 3.2, we draw a circle centered at $p_{1}$ with radius $r=d_{\min }\left(p_{1}, p_{B}\right)$ to further narrow down the feasible region of node $A$, which is

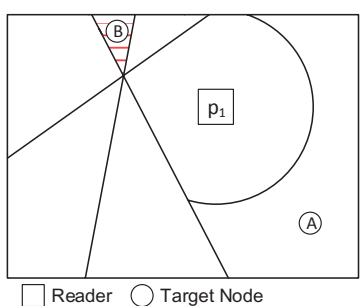

(a) Feasible Regions after Three Stops of the Charger

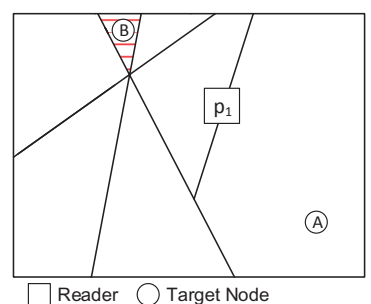

(b) Feasible Regions after the Convex Fix
Fig. 6. Inter-area Division

shown in Figure 6(a). Note that although the original feasible regions after Inter-area Division may not remain to be convex polygons, for the ease of analysis, we can replace them by their corresponding convex hulls, which are good approximations of the original shape (see Figure 6(b)).

\section{EXTENDED TOC DESIGN}

Based on the ideas of Basic TOC, in this section we show how to localize the target nodes more effectively by planning the charger stop positions. We first present an optimal solution for the scenario where single node is required to be localized. Then we extend the solution to solve the general multi-sensor scenario with a guaranteed approximate ratio.

\section{A. Optimal Region Cutting for One Node}

In this part, we introduce an optimal cutting algorithm which divides a feasible region with a single line. The charger will be able to decide the optimal stop sequence with this algorithm. Moreover, the results will provide insights to develop the solution for the more general scenario with multiple nodes.

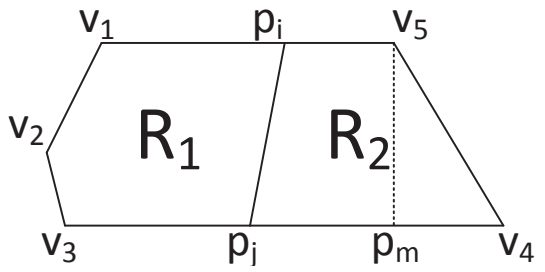

Fig. 7. Original Feasible Region $R$

1) Problem Definition: Consider an original feasible region $R$ and the set of its vertices $V_{R}=\left\{v_{1}, v_{2}, v_{3}, v_{4}, v_{5}\right\}$ as shown in Figure 7. $d(R)$ represents one diameter among the feasible region $R$. After another stop of the charger, one additional perpendicular bisector can be generated as the cutting line $l=$ $\left(p_{i}, p_{j}\right)$ where $p_{i}$ and $p_{j}$ are intersections with the edges of $R$. And this perpendicular bisector divides the region $R$ into two sub-regions $R_{1}$ and $R_{2}$. Since the longest diameter of $R_{1}$ and $R_{2}$ is positively related to the localization error, the optimal cutting problem aims to find the cutting strategy $p_{i}$ and $p_{j}$ so as to minimize the longest diameter of the resultant sub-regions. Mathematically, the problem can be written as

$$
\operatorname{Min}\left\{E_{\max }\right\}
$$


where $E_{\max }$ is termed as the Maximal Localization Error and

$$
E_{\max }=\operatorname{Max}\left\{d_{\max }\left(R_{1}\right), d_{\max }\left(R_{2}\right)\right\}
$$

2) Preliminaries: In order to transform the problem into a more concrete and simplified form, we introduce the following lemma and its corollary.

Lemma 4.1: With optimal cutting, the maximal localization error can only be achieved by one pair of vertices at either $R_{1}$ or $R_{2}$. Moreover, such a pair of vertices cannot be two neighboring vertices of the original region $R$.

We omit the proof of Lemma 4.1 due to space constraint (similarly hereinafter). Through Lemma 4.1, we can prove that one end of the longest distance among two cutting regions must be the cutting point (i.e. $p_{i}$ or $p_{j}$ ). Then Equation 2 can be reduced as:

$$
E_{\max }=\operatorname{Max}\left\{d_{\max }\left(p_{i}, V_{R}\right), d_{\max }\left(p_{j}, V_{R}\right)\right\}
$$

Formally, we have Corollary 4.2 as

Corollary 4.2: The optimal cutting problem is equivalent to the problem of finding a point $p_{i}$ that minimizes $d_{\max }\left(p_{i}, V_{R}\right)$.

Therefore, for minimizing the longest distance of the two cutting regions, we only need to find a point $p_{i}$ on edges of $R$, which has the minimal distance to the furthest vertex.

3) Algorithm Design: Intuitively, there are an infinite amount of points on the edges of $R$. To reduce the search space for $p_{i}$, firstly we introduce Theorem 4.3 which makes our optimal cutting algorithm practical.

Theorem 4.3: For the original region $R$, we define two sets of points

1) For any pair of vertices of $R$, such as $v_{i}$ and $v_{j}$, define $\left\{p_{i j}\right\}$ as the set of points which belong to the edges of $R$ and satisfy $d\left(p_{i j}, v_{i}\right)=d\left(p_{i j}, v_{j}\right)$. This means points in $\left\{p_{i j}\right\}$ have the same distance between vertex $v_{i}$ and $v_{j}$.

2) For any edge $l\left(v_{i}, v_{j}\right)$, define $\left\{p_{m}\right\}$ as the set of points, which belong to the edge $l\left(v_{i}, v_{j}\right)$ and satisfy $l\left(p_{m}, v_{k}\right) \perp$ $l\left(v_{i}, v_{j}\right)$, where $v_{k} \in V_{R}$ and $v_{k} \neq v_{i}, v_{j}$. This means points in $\left\{p_{m}\right\}$ are the perpendicular foots of any vertex on the edges of $R$.

Then $\operatorname{Min}\left\{d_{\max }\left(p_{i}, V_{R}\right)\right\}$ must be among the finite set composed of the above two kinds of points.

Based on Theorem 4.3, to find the optimal cutting, we first generate all candidate points $p_{i j}$ and $p_{m}$ which either satisfies $d\left(p_{i j}, v_{i}\right)=d\left(p_{i j}, v_{j}\right)$ or $l\left(p_{m}, v_{k}\right) \perp l\left(v_{i}, v_{j}\right)$ on every edge of $R$. We then compute the distance between each candidate point and vertices of $R$. After we put the point with the minimal value of maximal distance to vertices of $R$ on each edge into set $L$. When the algorithm terminates, the line which connects points in $L$ with the two minimal values is the optimal cutting line. Then the optimal charger stop position can be obtained through the fact that the optimal cutting line should be the perpendicular bisector of the optimal stop position and the previous stop position.
4) Complexity Analysis: Denote the number of vertices of $R$ as $n_{v}$ and the number of edges of $R$ as $n_{e}$, we have $n_{v}=n_{e}=$ $\left|V_{R}\right|$. Since $\left\{p_{i j}\right\}$ and $\left\{p_{m}\right\}$ are non-unique among different edges of $R$, we need to iterate through all edges in $R$ in the optimal algorithm and consequently there are $n_{v}$ choices. For each edge, we have at most $\left(n_{v}{ }^{2}\right)+\left(n_{v}-2\right)$ candidate points and we have to compute $d_{\max }\left(p, V_{R}\right)$ for $\left[\left(n_{v}{ }^{2}\right)+\left(n_{v}-2\right)\right] \cdot n_{v}$ times. Therefore, the overall algorithm complexity is $O\left(n_{v}^{4}\right)$.

5) Algorithm Performance and Insights: To evaluate the performance of the optimal cutting algorithm, we conduct the simulation where the charger chooses stop positions by the optimal cutting algorithm, and then compare the performance with Basic TOC where the charger stops randomly.

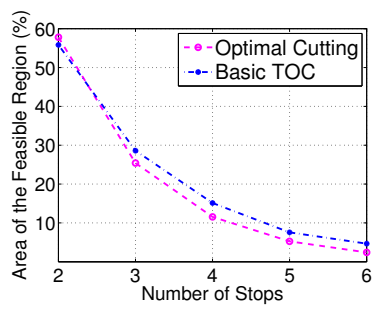

(a) Area of the Feasible Region

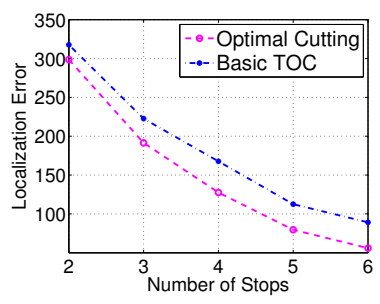

(b) Localization Error
Fig. 8. Optimal Cutting vs. Basic TOC (Single Node)

From Figure 8 we can see that both the area of the feasible region and localization error decrease with the increasing number of stops of the charger. In addition, the optimal cutting algorithm outperforms the random stop of the charger in Basic TOC both in terms of the feasible region area and localization error. Table I summarizes the percentage improvement of the optimal cutting algorithm in localization error compared with Basic TOC. For example, after six stops of the charger, the localization error decreases by $37.31 \%$.

TABLE I

LOCALIZATION ERROR IMPROVEMENT WITH OPTIMAL CUTTING

\begin{tabular}{c|c|c|c|c|c}
\hline \hline Number of Stops & 2 & 3 & 4 & 5 & 6 \\
\hline Improvement & $5.99 \%$ & $14.02 \%$ & $23.92 \%$ & $29.20 \%$ & $37.31 \%$ \\
\hline \hline
\end{tabular}

Furthermore, by examining the results of optimal cutting algorithm, we observe that the optimal cutting algorithm tends to cut off the longest diagonals of the feasible region. Specifically, the first or second longest diagonals are always being cut off for all simulation runs. Such insights allow us to design a more efficient approximation algorithm with performance guarantees in the next section.

\section{B. Cutting for Multiple Nodes}

The optimal cutting algorithms for the single node case divides the feasible region with minimized maximal localization error under the polynomial complexity. However, if the multisensor scenario is taken into consideration, we can no longer only focus on the feasible region of one node. Moreover, the Inter-area Division makes the computation even more complicated as the overall complexity of the optimal single cut solution is already $O\left(n_{v}^{4}\right)$. To handle this problem, in this part 
we firstly extend the optimal region cutting algorithm for one node to an approximation algorithm which is proved to be more general. Then inspired by the approximation solution for the single node case, we introduce an approximation solution for the multi-node scenario with a provable approximation ratio.

1) Extension of the Optimal Design: The main idea of our approximation algorithm is that by cutting more longer diagonal lines of the original polygon, we are more likely to minimize the maximal distance of two sub-divided regions.

The process of the approximation algorithm is explained as follows. Firstly we enumerate all pairs of vertices of the original polygon, calculate and sort diagonal lines between each pair of node in the non-increasing order. Then we iterate through the sorted diagonal lines and check whether there exists a line that intersects with all previous longest diagonals. To obtain a line that intersects a set of diagonal lines, we utilize a StabbingLineSegments $(L)$ function, which is a well-studied topic in computational geometry [7], [14]. The function returns false if the line does not exist. Essentially, this algorithm tries to continuously cut longest diagonal lines until it can not cut any more. Once the algorithm terminates, we can decide the corresponding charger stop position which could generate a perpendicular bisector that intersects the top $|L|$ longest lines within region $R$.

Given the number of vertices, $n_{v}$, the computation complexity of enumerating and sorting is $O\left(n_{v}^{3} \lg n_{v}\right)$. A widely accepted computation complexity for finding stabbing lines from a set of lines is $\Theta(n l g n)$ [7] where $n$ is the number of lines.

To quantitatively analyze the performance of our proposed approximation algorithm, we have the following theorem.

Theorem 4.4: Let $l_{\text {opt }}$ be the optimal dividing line calculated from the optimal cutting algorithm and $\left\{l_{a p p}\right\}$ be an infinite set of all feasible lines calculated from the approximation algorithm. We have $l_{\text {opt }} \in\left\{l_{\text {app }}\right\}$.

The insight of Theorem 4.4 is that the result of the optimal cutting algorithm is a special case among the approximation results. Based on Theorem 4.4, we have $\operatorname{Min}\{L\} \geq$ $\operatorname{Min}\left\{E_{\max }\right\} \geq \operatorname{Max}\{D\}$. In other words, we expand the optimal result in section IV-A to the range between $\operatorname{Min}\{L\}$ and $\operatorname{Max}\{D\}$. Consequently, the approximation ratio of our algorithm $\rho=\operatorname{Max}\{\operatorname{Min}\{L\}\} / E_{\text {opt }} \leq \operatorname{Min}\{L\} / \operatorname{Max}\{D\}$.

2) Cutting for Multiple Nodes: In multi-sensor scenario, it is intrinsically difficult to obtain the optimal stop position of the charger due to the high complexity in both spatial and temporal dimensions. Thus we focus on a heuristic design which extends the approximation algorithm for single node with guaranteed localization performance.

The main idea of the algorithm for the multi-node case is to cut more diagonals of all feasible regions every time with joint consideration of all nodes. Denote the number of nodes as $N$, we first calculate distances among vertices of all nodes and sort them in set $D$. After that, we gradually put elements of $D$ into set $L$ according to the descending order of its length.
Therefore, it can be guaranteed that the longest $|L|$ lines among all feasible regions will be cut at each step.

In this way, we approximately minimize the maximal distance among all target nodes in the network. It can be proved that the approximation ratio of the multi-node algorithm is $\rho=\operatorname{Min}\{L\} / \operatorname{Max}\{D\}$. In other words, our approximation algorithm guarantees that, after cutting, the longest distance among feasible regions will be less than $\rho$ times of the optimal solution.

Recall that in Basic TOC, we can draw $n-1$ perpendicular bisectors at the $n$-th stop of the charger. Therefore to generate the cutting line, we have $n-1$ candidate stop positions. To make full use of these perpendicular bisectors, among these candidates we choose the optimal stop position which minimizes the average localization error.

\section{Discussion}

In previous sections, we introduce both basic TOC and advanced TOC under the assumption that the charging time is strictly negatively correlated with the charging distance. In this section, we discuss the impact of occasional charging time flips in TOC design and elaborate solutions on dealing with such cases.

Typically, the flips of charging times are mainly due to two major reasons. The first reason is the radio irregularity and interference in wireless charging. Similar to wireless communications, such radio irregularity is caused by the nonisotropic properties of the propagation media and the heterogeneous properties of devices [32]. The second reason is the measurement errors. Such errors are caused by measurement noises and random wireless communication errors such as packet loss and collisions. Both of above two reasons could lead to the change of instantaneous charging powers on WRSN nodes and cause the flips of charging times.

However, since TOC compares the charging time differences among individual nodes instead of instantaneous charging power, it is more robust and resilient to instantaneous charging power fluctuations caused by various factors. For example, through our empirical measurement, the coefficient of variation of charging power is four times as high as that of charging time.

To deal with occasional potential charging time flips, in our design, we propose a simple yet effective solution. Assume the charging time of node $A$ is $T_{i}$ and $T_{j}$ respectively when the charger stops at location $p_{i}$ and $p_{j}$. We divide the feasible region of node $A$ only when $\left|T_{i}-T_{j}\right|>\frac{\varepsilon}{\lg \left(\min \left\{T_{i}, T_{j}\right\}\right)}$ in Section III-B and Section III-C. Rationale of the equation is based on two observations: i) the probability of flip is low if there exists a relatively large gap between charging times of two nodes; ii) for a fixed difference between charging times of two pairs of nodes, flip is more likely to happen in the pair of nodes which are closer to the charger. We term $\varepsilon$ as the unreliability threshold and empirically choose its value for different WRSN platforms. For example, for our WRSN testbed used in Section 
VI-A, we set $\varepsilon$ as 0.2 based on the offline measurement results in Figure 3. Essentially, TOC with a larger $\varepsilon$ is able to tolerate severer charging power fluctuations as well as greater device diversity whereas leads to slower localization of the nodes. We omit the evaluation results due to the space constraints.

\section{PERformance EVAluation}

In this section, we first evaluate the performance of TOC through experiments. Large-scale simulations are further proposed in Section VI-C and Section VI-D.

\section{A. Experimental Evaluation}

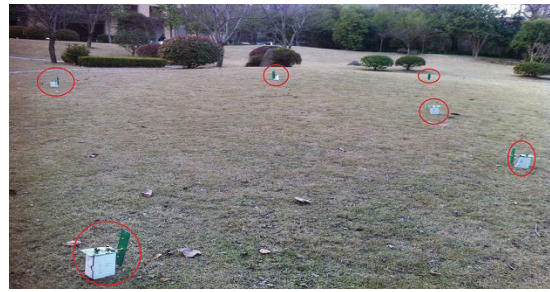

Fig. 9. Experimental Area

We firstly evaluate TOC design on our WRSN testbed with six rechargeable sensor nodes. During the experiment, nodes are randomly distributed in an area of $15 \mathrm{~m} * 15 \mathrm{~m}$ (shown in Figure 9) where a RFID reader (charger) can stop at different locations to charge all six nodes. At each stop position, the charger wirelessly charges all the nodes to a certain voltage and records the time of charge for each node. Without any prior knowledge, we randomly choose the first two stop positions, then calculate the following stop positions based on our extended TOC design in Section IV-B. The localization process terminates when the total number of stops reaches 5 .

Figure 10 shows how the feasible regions of all nodes evolves along with each stop of the charger and the summarized localization results are listed in Table II.

TABLE II

EXPERIMENTAL RESULTS

\begin{tabular}{c|c|c|c|c}
\hline \hline Number of Stops & 2 & 3 & 4 & 5 \\
\hline Area of Feasible Region (\%) & $47.97 \%$ & $20.51 \%$ & $10.84 \%$ & $2.78 \%$ \\
\hline Mean Localization Error (m) & 3.82 & 2.95 & 1.82 & 0.81 \\
\hline \hline
\end{tabular}

From Table II we can see that after three stops of the charger, $80 \%$ original area is excluded and the mean localization error decreases to less than $3 \mathrm{~m}$. After 5 stops of the charger, the average localization error has been reduced to $0.81 \mathrm{~m}$. Specifically, Figure 11 further shows the feasible regions and localization errors for each nodes after 5 stops of charger. From Figure 11 we find that all 6 nodes achieve good localization performance at around $1 \mathrm{~m}$ and there is a small variation among different nodes. Since almost no flips of charging times can be observed on our testbed, we directly set the unreliability threshold $\epsilon$ as 0 during the experiment. In fact, if we increase $\epsilon$ from 0 to 1 , around $17 \%$ cutting probabilities will be filtered which will increase the localization error instead.

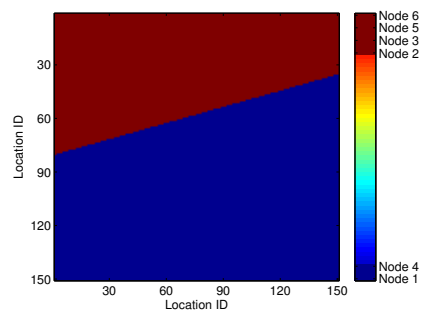

(a) Stop 2



(c) Stop 4

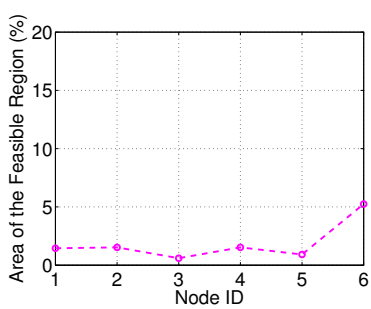

(a) Area of Feasible Region (\%)

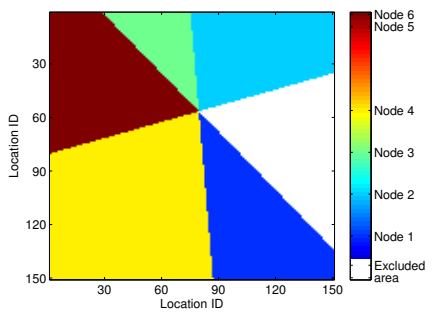

(b) Stop 3



(d) Stop 5



(b) Localization Error (m)
Fig. 11. Localization Results among Different Nodes

\section{B. Simulation Settings}

TABLE III

DeFault Simulation PARAMETERS

\begin{tabular}{|c|c|}
\hline Parameters & Description \\
\hline Field Area & $100 \times 100$ (Grid Unit) \\
\hline Number of Stops & 6 \\
\hline Number of Target Nodes & 100 \\
\hline Target Node Distribution & Uniform Distribution \\
\hline Statistics & Feasible Region; Localization Error \\
\hline Random Seed & 100 runs \\
\hline
\end{tabular}

In addition to experiment evaluation, we conduct large-scale simulation to evaluate the performance of the Basic TOC, followed by the performance comparison between the Basic TOC and the Extend TOC. Default simulation parameters are shown in Table III. Note that due to the positive relationship between the charging distance and charging time, we do not need to specify charging model and perform cutting only based on binary comparison results of each pair of nodes. We adopt percentage area of the feasible region and the localization error as two metrics to evaluate localization performance. The percentage of the feasible region refers to the ratio of segmented area after each stop over the area of the original feasible region. The localization error is defined as the distance 
between the estimated position and the real location of the sensor. In addition, we also calculate the standard deviation of localization error among different sensors.

\section{Performance of Basic TOC}

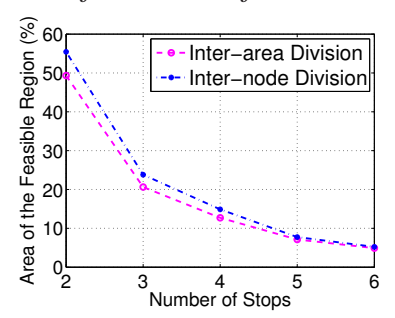

(a) Area of the Feasible Region

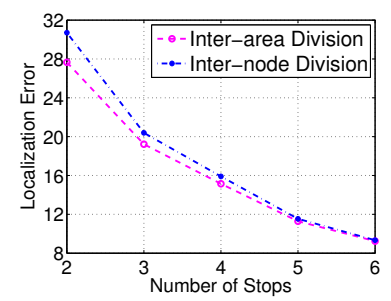

(b) Mean Localization Error
Fig. 12. Localization Result of the Basic TOC (Number of Nodes $=100$ )

TABLE IV

DECREASE OF LOCALIZATION ERROR

\begin{tabular}{c|c|c|c|c|c}
\hline \hline Number of Stops & 2 & 3 & 4 & 5 & 6 \\
\hline Gain (\%) & $9.90 \%$ & $5.74 \%$ & $4.72 \%$ & $2.17 \%$ & $1.07 \%$ \\
\hline \hline
\end{tabular}

TABLE V

DECREASE OF STANDARD DEVIATION OF LOCALIZATION ERROR

\begin{tabular}{c|c|c|c|c|c}
\hline \hline Number of Stops & 2 & 3 & 4 & 5 & 6 \\
\hline Gain (\%) & $21.91 \%$ & $18.03 \%$ & $26.00 \%$ & $24.42 \%$ & $26.17 \%$ \\
\hline \hline
\end{tabular}

In order to show the effectiveness of Basic TOC, we first compare the localization performance of purely Inter-node Division approach with Complete Basis TOC, which combines Inter-node Division and Inter-area Division. From Figure 12(a) and Figure 12(b) we can see that Complete Basic TOC does achieve better performance in terms of both the area of feasible region and the mean localization error. Table IV summarize the percentage gain of the Inter-area Division. From Table IV, we find that the percentage gain of the Inter-area Division decreases as the charger stops for more times. This is because the area of the feasible regions of nodes decrease quickly along with the number of charger stops which makes the inter-area dividing happen less frequently.

In Table V, we examine the gain in standard deviation of localization error among different nodes. We find that the Complete Basic TOC provides a significantly smaller standard deviation than that of the purely Inter-node Division. For example after the fourth stop of the charger, the standard deviation gain is as high as $26.0 \%$. Results of Table $\mathrm{V}$ well support the effectiveness of Inter-area Division which utilizes the well positioned nodes to help localize other related nodes so that the error deviation through the network can be kept at a lower level.

\section{Extended TOC vs. Basic TOC}

This section illustrates performance of the Extended TOC over the Basic TOC. To qualitatively analyze localization performance, we also plot the optimal results through exhaustive search with granularity $g=10$ grid unit, which at each step selects the charger stop position for minimizing the mean localization error over the network.

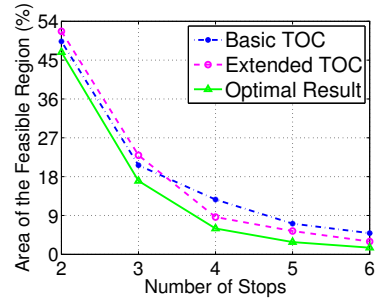

(a) Area of the Feasible Region

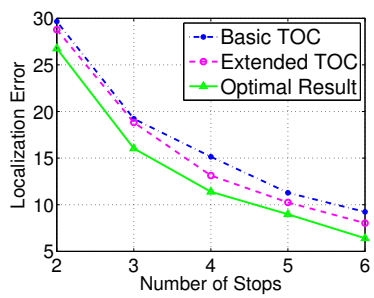

(b) Mean Localization Error
Fig. 13. Extended TOC vs. Basic TOC (Number of Nodes $=100$ )

In Figure 13(a) and Figure 13(b), by optimizing the charger stop positions, the Extended TOC is able to reduce both the area of feasible regions and mean localization errors. And the localization error decreases monotonically to around $8 \mathrm{~m}$ after 6 stops. Detailed percentage gain of the mean localization error of both Extended TOC and the optimal results are shown in Table VI. For example after 4 stops of the charger, mean localization error of the Extend TOC is $13.33 \%$ less than the result of the Basic TOC (13.13m vs $15.15 \mathrm{~m})$ and the optimal result owns a $24.84 \%$ decrease (11.39 vs $15.15 \mathrm{~m}$ ). Comparing with the optimal results, we find that the Extended TOC offers a fairly good localization performance with large computation complexity degradation.

TABLE VI

DECREASE OF LOCALIZATION ERROR

\begin{tabular}{c|c|c|c|c|c}
\hline \hline Number of Stops & 2 & 3 & 4 & 5 & 6 \\
\hline Extended TOC (\%) & $2.97 \%$ & $2.09 \%$ & $13.33 \%$ & $9.27 \%$ & $13.16 \%$ \\
\hline Optimal Result (\%) & $9.81 \%$ & $16.58 \%$ & $24.84 \%$ & $20.47 \%$ & $30.70 \%$ \\
\hline \hline
\end{tabular}

\section{RELATED WORK}

Many work has been proposed to localize nodes in wireless sensor networks. Based on the underlying localization techniques, there are mainly two types of methods: rangebased localization and range-free localization. Range-based localization method such as GPS, AOA [18], Sweeps [8], SRIPS [5] and ArrayTrack [27] measure point-point distances or angles among sensor nodes and/or anchor nodes to compute per-node position. Although they tend to offer precise locations of nodes, they incur unfavorable costly additional hardware or environment profiling. Range-free localization methods [6], [21], [28], [31], on the other hand, localize nodes based on their connectivity information or simple sensing of their relative positions. However, such kinds of localization systems either need several anchor nodes or precise event distributions which make them less effective for practical deployment.

Another category of localization algorithm is based on received signal strength indication (RSSI) [17], [30]. However due to the inherent variability in wireless signal propagation characteristics and the presence of severe multipath fading with multiple reflections, the relationship between RSSI and 
distance is extremely hard to model, limiting the accuracy of both model-based methods and fingerprinting-based methods.

Wireless power transfer technology has been adopted in many existing WRSNs. One representative example is Wireless Identification and Sensing Platform (WISP) which harvest energy from the off-the-shelf commercial RFID reader [22]. After being first proposed in 2008, there have been many works on WISP based WRSN [9], [26].

Despite the diversity of work has been done on both localization of sensor nodes and wireless rechargeable sensor networks, little work has been proposed on localization in WRSN. In this paper, we adopt the fundamental charging principle and propose a Time of Charge based localization method. Our design is compatible with most wireless rechargeable sensor networks as well as wireless energy harvesting technologies. In addition, it avoids the limitations of range-based, rangefree and RSSI-based localization methods and does not rely on additional hardware, anchors or generated events.

\section{CONCLUSION}

In this paper, we study the problem of how to use the Time of Charge sequences of wireless rechargeable sensor nodes to localize themselves. We first identify the fundamental relationship between charging time and distance between nodes and the charger. Based on this principle, we propose two localization methods, i.e., Basic TOC and Extended TOC. In the Basic TOC, the charger stops randomly in the field to charge nodes and divides their feasible regions iteratively. In the Extended TOC, we further optimize the charger stop positions based on the estimated sensor locations so as to achieve higher localization accuracy. To verify our designs, we perform detailed performance evaluations through analysis and large-scale simulations. To the best of our knowledge, it is the first work designed for localization in wireless rechargeable sensor network by purely using the Time of Charge sequences.

\section{ACKNOWLEDGMENT}

The work was supported in part by NSFC under Grants 61222305 and 61228302, 111 Program under Grant B07031, National Program for Special Support of Top-Notch Young Professionals, scholarship funded by China Scholarship Council, Grant SUTD-ZJU/RES/03/2011, TELAMON-IRPS and NSF under grant CNS-0845994.

\section{REFERENCES}

[1] A. Abdulla, H. Nishiyama, J. Yang, N. Ansari, and N. Kato. HYMN: A Novel Hybrid Multi-Hop Routing Algorithm to Improve the Longevity of WSNs. IEEE Transactions on Wireless Communications, 11(7):25312541, 2012.

[2] European Retailer Throttleman Improves Supply Chain with RFID. http: //www.alientechnology.com/docs/CS_Throttleman.pdf.

[3] D. J. Bijwaard, W. A. van Kleunen, P. J. Havinga, L. Kleiboer, and M. J. Bijl. Industry: Using Dynamic WSNs in Smart Logistics for Fruits and Pharmacy. In ACM SenSys, 2011.

[4] X. Cao, J. Chen, Y. Zhang, and Y. Sun. Development of an integrated wireless sensor network micro-environmental monitoring system. ISA transactions, 47(3):247-255, 2008.
[5] B. Dil and P. J. M. Havinga. Stochastic radio interferometric positioning in the $2.4 \mathrm{GHz}$ range. In ACM SenSys, pages 108-120, 2011.

[6] L. Doherty, K. pister, and L. El Ghaoui. Convex position estimation in wireless sensor networks. In IEEE INFOCOM, 2001.

[7] H. Edelsbrunner, H. A. Maurer, F. P. Preparata, A. L. Rosenberg, E. Welzl, and D. Wood. Stabbing line segments. BIT, 22(3):274-281, 1982.

[8] D. K. Goldenberg, P. Bihler, Y. R. Yang, M. Cao, J. Fang, A. S. Morse, and B. D. O. Anderson. Localization in sparse networks using sweeps. In ACM MOBICOM, pages 110-121, 2006.

[9] J. Gummeson, S. S. Clark, K. Fu, and D. Ganesan. On the Limits of Effective Hybrid Micro-energy Harvesting on Mobile CRFID Sensors. In ACM MobiSys, 2010.

[10] L. He, Z. Yang, J. Pan, L. Cai, and J. Xu. Evaluating service disciplines for mobile elements in wireless ad hoc sensor networks. In IEEE INFOCOM, 2012.

[11] S. He, J. Chen, F. Jiang, D. K. Y. Yau, G. Xing, and Y. Sun. Energy provisioning in wireless rechargeable sensor networks. In IEEE INFOCOM, 2011.

[12] T. He, C. Huang, B. M. Blum, J. A. Stankovic, and T. Abdelzaher. RangeFree Localization Schemes for Large Scale Sensor Networks. In ACM MobiCom, pages 81-95, 2003.

[13] B. Karp and H. Kung. GPSR: Greedy Perimeter Stateless Routing for Wireless Networks. In ACM MobiCom, 2000.

[14] M. J. Katz, J. S. B. Mitchell, and Y. Nir. Orthogonal segment stabbing. Comput. Geom., pages 197-205, 2005.

[15] Y. Kim, R. Govindan, B. Karp, and S. Shenker. Geographic Routing Made Practical. In NSDI, 2005.

[16] G. Liu, W. Yu, and Y. Liu. Resource Management with RFID Technology in Automatic Warehouse System. In IEEE IROS, 2006.

[17] D. Madigan, E. Einahrawy, R. Martin, W.-H. Ju, P. Krishnan, and A. Krishnakumar. Bayesian indoor positioning systems. In IEEE INFOCOM, 2005.

[18] D. Niculescu and B. R. Badrinath. Ad Hoc Positioning System (APS) Using AOA. In IEEE INFOCOM, 2003.

[19] T. C. Poon, K. L. Choy, H. K. H. Chow, H. C. W. Lau, F. T. S. Chan, and K. C. Ho. A RFID Case-based Logistics Resource Management System for Managing Order-picking Operations in Warehouses. Expert Syst. Appl., 36(4):8277-8301, 2009.

[20] B. Ransford, J. Sorber, and K. Fu. Mementos: System Support for LongRunning Computation on RFID-Scale Devices. In ASPLOS, 2011.

[21] K. Römer. The Lighthouse Location System for Smart Dust. In ACM MobiSys, 2003.

[22] A. P. Sample, D. J. Yeager, P. S. Powledge, A. V. Mamishev, and J. R. Smith. Design of an RFID-Based Battery-Free Programmable Sensing Platform. IEEE T. Instrum. Meas., 57(11):2608-2615, 2008.

[23] A. Savvides, C. Han, and M. Srivastava. Dynamic Fine-Grained Localization in Ad-Hoc Networks of Sensors. In ACM MobiCom, 2001.

[24] Y. Shu, Y. Gu, and J. Chen. Sensory-data-enhanced authentication for RFID-based access control systems. In IEEE MASS, pages 236-244, 2012.

[25] E. W. Weisstein. Plane division by lines. mathworld.wolfram.com.

[26] First workshop on Wirelessly Powered Sensor Networks and Computational RFID. http://seattle.intel-research.net/wisp/summit/.

[27] J. Xiong and K. Jamieson. ArrayTrack: A Fine-Grained Indoor Location System. In USENIX NSDI, 2013.

[28] Q. Xu, A. Gerber, Z. M. Mao, and J. Pang. AccuLoc: Practical localization of performance measurements in $3 \mathrm{G}$ networks. In $A C M$ MobiSys, 2011.

[29] L. Yang, J. Han, Y. Qi, and Y. Liu. Identification-free Batch Authentication for RFID Tags. In IEEE ICNP, 2010.

[30] M. Youssef and A. K. Agrawala. The Horus WLAN location determination system. In ACM MobiSys, 2005.

[31] Z. Zhong and T. He. Achieving range-free localization beyond connectivity. In ACM SenSys, pages 281-294, 2009.

[32] G. Zhou, T. He, S. Krishnamurthy, and J. A. Stankovic. Impact of Radio Irregularity on Wireless Sensor Networks. In ACM MobiSys, 2004.

[33] Y. Zhuang, J. Pan, Y. Luo, and L. Cai. Time and Location-Critical Emergency Message Dissemination for Vehicular Ad-Hoc Networks. IEEE Journal on Selected Areas in Communications, 29(1):187-196, 2011. 